\title{
Distinct clinicopathologic and radiological manifestations of skin, lung, and muscle diseases in dermatomyositis patients positive for anti-aminoacyl tRNA synthetase antibodies
}

Hiroko Fukamatsu a Yoji Hirai a , Tomoko Miyake a, Tatsuya Kaji a , Shin Morizane a , Emi Yokoyama a , Toshihisa Hamada a, Takashi Óono b, Yoshinobu Koyama c, Śeiichiro Norikane d, Keiji Iwatsúki a

a Departments of Dermatology, Okayama University Graduate School of Medicine, Dentistry, and Pharmaceutical Sciences, Okayama.

b Japanese Red Cross Okayama Hospital, Okayama,

c Department of Rheumatology, Japanese Red Cross Okayama Hospital, Okayama,

${ }^{d}$ Department of Radiology, Japanese Red Cross Himeji Hospital, Hyogo

\section{[Background]}

- Patients with antisynthetase syndrome (ASS), which is diagnosed by the presence of an anti-aminoacyl tRNA synthetase (ARS) antibody, may constitute a unique subtype of dermatomyositis patients.

- So far, eight anti-ARS antibodies (Jo-1, PL-7, PL-12, EJ, OJ, KS, Zo, and $\mathrm{Ha}$ ) have been found.

[Aim of the present study]

- To examine the autoantibody profiles of patients with dermatomyositis, and compare the results with clinical manifestations between the ASS and non-ASS group.

\section{[Patients]}

- Fifty-nine patients with DM who visited our clinics from 2008 to 2016.

- Fifty-two patients met the criteria for the classification tree model by the International Myositis Classification Criteria Project (IMCCP)*. - The remaining 7 patients include:

4 "possible" cases of Bohan and Peter's criteria,

1 "probable" case,

2 cases of amyopathic DM with ILD.

[Methods]

- ELISA kit: MESACUP anti-ARS test (Jo-1, PL-7, PL-12, EJ, and KS)

- All sera were examined for antibodies to TIF1- $\gamma$, MDA5 and Mi-2.

- Evaluation of cutaneous and systemic symptoms, including radiological images of interstitial lung disease (ILD) and myositis, in a blind fashion.

- The results were analyzed in relation to serum levels of KL-6, CK, ALD, and ALD/CK ratios between the ASS and non-ASS groups.

[Result 1] Antibody profile of the ASS and non-ASS groups

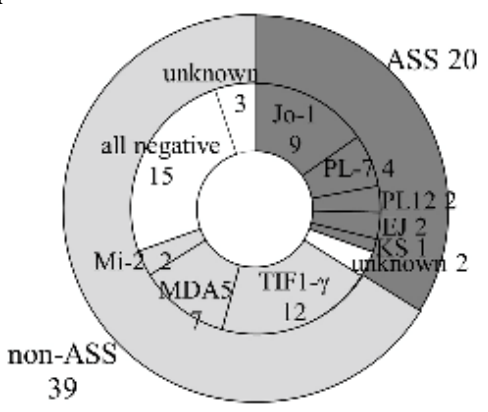

[Result 2] Cutaneous and systemic symptoms

\begin{tabular}{|c|c|c|c|}
\hline Clinical symptoms and signs & ASS & non-ASS & p value \\
\hline Age of onset, $y$ & $51.7 \pm 14.7$ & $59.7 \pm 14.5$ & 0.043 \\
\hline Sex ratio, $M: F$ & $7: 13$ & $13: 26$ & 1.00 \\
\hline Heliotrope rash & $5 / 20(25 \%)$ & $20 / 39(51 \%)$ & 0.094 \\
\hline ottron's papule & $12 / 20(60 \%)$ & $33 / 39(85 \%)$ & 0.053 \\
\hline Erythema on the extensor surface of extremities & $15 / 20(75 \%)$ & $27 / 39(69 \%)$ & 0.77 \\
\hline Mechanic's hand & $14 / 20(70 \%)$ & $13 / 39(38 \%)$ & 0.012 \\
\hline Shawl-sign/V-sig & $3 / 20(15 \%)$ & $20 / 39(51 \%)$ & 0.010 \\
\hline & $3 / 20(15 \%)$ & $4 / 39(10 \%)$ & 0.68 \\
\hline Per & $11 / 20(55 \%)$ & $28 / 39(72 \%)$ & 0.25 \\
\hline Poiki & $0 / 20(0 \%)$ & $7 / 39(18 \%)$ & 0.08 \\
\hline Scre & $3 / 20(15 \%)$ & $11 / 39(28 \%)$ & 0.34 \\
\hline Pro & $10 / 20(50 \%)$ & $26 / 39(67 \%)$ & 0.26 \\
\hline $\mathrm{Mu}$ & $10 / 20(50 \%)$ & $2 S / 39(69 \%)$ & 0.59 \\
\hline rum & $12 / 12(100 \%)$ & $18 / 26(69 \%)$ & 0.0022 \\
\hline th -itia & $13 / 20(65 \%)$ & $9 / 39(23 \%)$ & 0.0037 \\
\hline Elevated serum CRP level & $16 / 20(80 \%)$ & $15 / 39(38 \%)$ & 0.0029 \\
\hline Fever of over $38^{\circ} \mathrm{C}$ & $8 / 16(60 \%)$ & $6 / 39(14 \%)$ & 0.019 \\
\hline Incidence $c$ & $19 / 20(95 \%)$ & $17 / 39(44 \%)$ & 0.0001 \\
\hline Incidence of malignancy & $4 / 20(20 \%)$ & $13 / 39(33 \%)$ & 0.37 \\
\hline History of collagen disease in relatives & $6 / 20(30 \%)$ & $0 / 39(0 \%)$ & 0.000 \\
\hline
\end{tabular}

[Result 3] Incidence of ILD and CT image

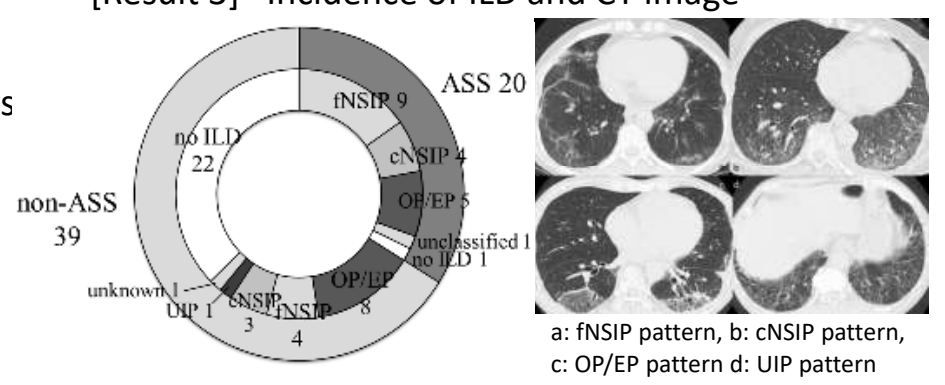

[Result 4] Myositis pattern and serum ALD levels

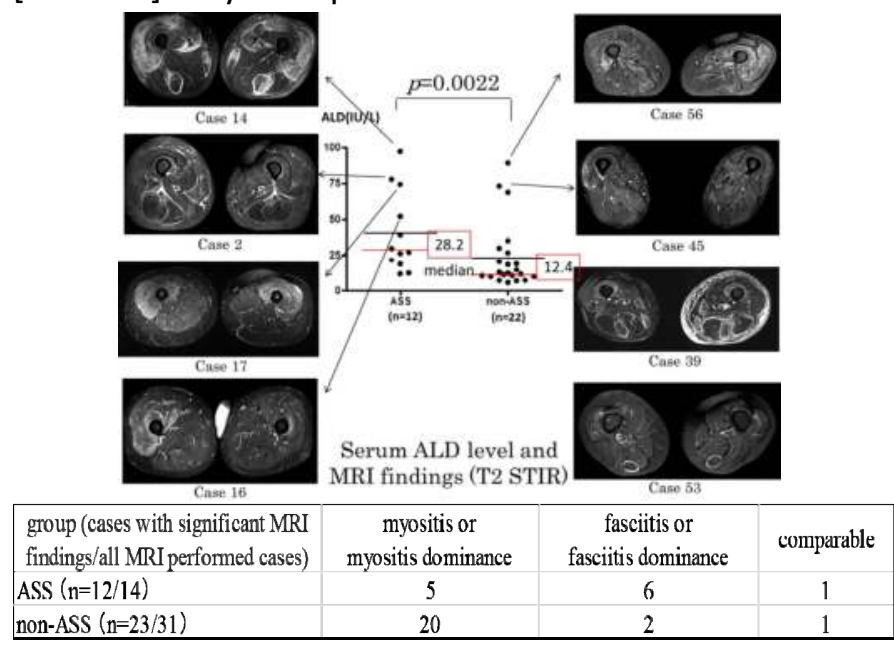

[Conclusion]

Patients with ASS have a higher incidence of mechanic's hands, shawl sign, systemic inflammation, ILD, and inflammatory myopathy associated with fasciitis, and elevated serum levels of ALD.

[References]

1. Mahler M, et al. Autoimmun Rev 2014; 13: 367-3712.

2. Lega JC, et al. Autoimmun Rev 2014; 13: 883-891.

3. Hamaguchi Y, et al. PLOS ONE 2013; 8: e60442.

COI: All authors have no $\mathrm{COI}$ to disclose. Acknowledgments: The present study was made possible by technical support by Masahiro Hashiguchi and Akihiko Murakami (MBL, Co., LTD). 